\section{PREGNANCY PLUS Epilepsy in pregnancy}

\author{
Torbjörn Tomson, ${ }^{1}$ Vilho Hiilesmaa²
}

'Department of Clinical Neuroscience, Karolinska Institutet, SE-17176 Stockholm, Sweden 2Department of Obstetrics and Gynaecology, Helsinki University, PL140, 00029 HYKS, Finland torbjorn.tomson@karolinska.se

BMJ 2007;335:769-3 doi:10.1136/bmj.39266.473113.BE occasional articles about how to manage a pre-existing medical condition during pregnancy
This is one of a series of

\section{This article explores the therapeutic problems that arise when a patient with epilepsy on treatment becomes pregnant and needs both effective seizure control and attention to the safety of her fetus}

Epilepsy is usually managed by neurologists or general practitioners. Managing epilepsy during pregnancy is a major therapeutic challenge, as the potential adverse effects of antiepileptic drugs on the fetus must be balanced against the maternal and fetal risks associated with uncontrolled seizures. The situation we describe is ideal as our patient told her neurologist of her plans to become pregnant (see Scenario box). In such cases, the patient can receive counselling and treatment changes can be put in place before conception, whereas in many cases the woman is already pregnant when she alerts her doctors. Switching from valproate to lamotrigine was successful in our patient, but the effects of such switches are unpredictable. Some patients will have relapses or an increase in the frequency of seizures, which may prompt trials of other drugs. Some women may need to revert to the original treatment to control seizuresin this case valproate at the lowest effective dosage. Any switches between antiepileptic drugs should be accomplished and assessed before conception to avoid

\section{Scenario}

A 33 year old woman had had occasional myoclonic jerks in the mornings since the age of 15 , usually after sleep deprivation. Two years later, after her first generalised tonic-clonic seizure, she was diagnosed with juvenile myoclonic epilepsy, an idiopathic generalised epilepsy. After her second tonic-clonic seizure she was prescribed valproate. During the next two years, she had a few more tonic-clonic seizures until the dosage of valproate was increased to $500 \mathrm{mg}$ twice daily. On this medication, she was free from seizures for 11 years and only had isolated myoclonic jerks-years apart-always after sleep deprivation.

Two years ago, her medication was changed from valproate to lamotrigine in response to her plans for pregnancy and the concern that valproate could be teratogenic. This conversion was uneventful. She has remained free from tonic-clonic seizures, but her myoclonic jerks have been slightly more frequent despite a lamotrigine dose of $150 \mathrm{mg}$ twice daily.

The pregnancy was planned together with her neurologist. She was followed more closely during pregnancy, with clinical check-ups and monitoring of lamotrigine plasma concentrations every second month. The dosage of lamotrigine was gradually increased to $250 \mathrm{mg}$ twice daily during the second half of pregnancy in response to an increased frequency of myoclonic jerks caused by a fall in lamotrigine plasma concentrations to less than half of the prepregnancy values. She had an uneventful delivery without seizures and gave birth to a healthy child. The lamotrigine dosage was gradually decreased to $150 \mathrm{mg}$ twice daily within three days of delivery.

\author{
Box 1 | Factors that may affect seizure control in \\ pregnancy and delivery \\ - Non-compliance with and withdrawal of drugs (often due \\ to fear of teratogenic effects) \\ - Alterations in pharmacokinetics of antiepileptic drugs \\ caused by gestation \\ - Pregnancy related hormonal changes \\ - Sleep deprivation in pregnancy; physical and mental \\ stress in labour
}

risks to the fetus induced by maternal seizures.

This article focuses on the management of women who have seizures before pregnancy and not those who have them for the first time during pregnancy, such as women who develop eclampsia.

\section{How common is epilepsy in pregnancy?}

Population based studies indicate a prevalence of epilepsy in pregnant women of up to $0.7 \%,{ }^{1}$ although register based studies have reported prevalences of $0.2-0.4 \% .^{2}{ }^{3}$ Women other than those with epilepsy take antiepileptic drugs, however, as they are increasingly being prescribed for psychiatric disorders and neuropathic pain disorders.

\section{Does pregnancy affect epilepsy?}

Pregnancy has no effect on seizure control in most women with epilepsy. Although population based studies indicate that symptoms deteriorate in $15-30 \%$ of women, they improve in a similar proportion of women. ${ }^{4-7}$ This could partly reflect random fluctuations. In a prospective international study of 1736 pregnancies, about $60 \%$ of women remained seizure free throughout pregnancy. ${ }^{8}$ Delivery and labour carry an increased risk, with 2-5\% of women with epilepsy having seizures at these times. ${ }^{8}$ Antiepileptic drugs may differ with respect to efficacy during pregnancy. Fewer women were seizure free on oxcarbazepine, and dose increases during pregnancy were more frequent with oxcarbazepine and lamotrigine. ${ }^{8}$ This may be related to changes in the pharmacokinetics of these drugs during pregnancy (box 1). ${ }^{9}$

\section{How do seizures affect pregnancy?}

Although the absolute risk is low, a larger than expected proportion of maternal deaths in the United Kingdom is caused by epilepsy. This may be accounted for by seizures in women who stop taking their drugs when 
they realise that they are pregnant. ${ }^{10}$ This illustrates the importance of effective treatment. While other seizure types have negligible effects, tonic-clonic seizures increase the pressure in the pregnant uterus, and may lead to trauma if the patient falls. They can also cause lactic acidosis, ${ }^{11}$ which is transferred to the fetus. ${ }^{12}$ However, recent reports suggest that the number of stillbirths in adequately treated women with epilepsy is similar to that in the background population. ${ }^{3}{ }^{13}$ There is no hard evidence of increased risk of obstetric complications, such as pre-eclampsia, premature delivery, or placental abruption. ${ }^{3} 1314$ The seizures or the epilepsy itself are unlikely to contribute greatly to the increased risk of birth defects reported for women with epilepsy. ${ }^{15}$

Epilepsy is not an indication for early induction of labour or elective caesarean section. Caesarean section is needed if frequent tonic-clonic seizures or other seizures greatly impair cooperation in the forthcoming labour and delivery. A caesarean delivery may be necessary if a generalised tonic-clonic seizure occurs during labour. Refractory status epilepticus in the third trimester of pregnancy could also be an indication for a caesarean. These are rare occurrences and most women with epilepsy have normal deliveries.

What are the risks to the fetus from antiepileptic drugs? Antiepileptic drugs have been associated with birth defects and impaired postnatal cognitive development. ${ }^{16}$ The risk of malformations is two to three times that expected in the general population with older generation drugs, such as phenobarbital, phenytoin, carbamazepine, and valproate. ${ }^{16} 17$ Treatment with more than one drug is associated with higher rates of birth defects. ${ }^{2}{ }^{18-22}$ The risk for monotherapy with valproate, ${ }^{2} 192023$ and probably other drugs, ${ }^{18}$ seems to be dose dependent.

The pattern of birth defects varies with the type of antiepileptic drug. Neural tube defects have been linked to the use of carbamazepine, and particularly valproate. ${ }^{17} \mathrm{~A}$ recent report suggests that lamotrigine increases the risk of oral clefts. ${ }^{24}$

The table summarises the evidence from recent large registries on the teratogenic effects of different antiepileptic drugs. Because teratogenic effects cannot be analysed in randomised controlled trials, the evidence comes from observational studies (at best class 2 level) and results must be interpreted with caution. These studies all indicate a greater risk for birth defects with valproate than with other antiepileptic drugs-rates range from $6 \%$ to $11 \%$ in children exposed to valproate (table). The North American epilepsy and pregnancy registry has also reported major malformations in $6.5 \%$ of 77 prospective pregnancies with phenobarbital as monotherapy, four times higher than in an external unexposed control group, but not significantly higher than three other antiepileptic drugs combined from the same registry. ${ }^{29}$ However, other retrospective and prospective cohort studies ${ }^{19-21}$ have not revealed higher malformation rates with phenobarbital than with carbamazepine. ${ }^{16}$

${ }^{17}$ Except for lamotrigine, data on pregnancy outcome for new antiepileptic drugs are too scarce to assess their teratogenicity. ${ }^{30}$

Data on effects of antiepileptic drugs on cognitive development in the offspring of mothers treated in pregnancy are less conclusive. ${ }^{31}$ However, a recent retrospective study from the UK suggests that valproate, in a dose dependent way, may be associated with significantly lower verbal IQ compared with carbamazepine and phenytoin or with unexposed children. ${ }^{10}{ }^{32} \mathrm{~A}$ similar trend, although not statistically significant, was found in two small population based prospective studies. ${ }^{33} 34$

These observational studies on pregnancy outcome need to be interpreted with caution as potential confounding factors have not been controlled for. Nevertheless, higher birth defect rates with valproate than with carbamazepine and the possibility of intellectual impairment call for caution in the use of valproate in pregnancy. Our patient was therefore switched to lamotrigine when planning her pregnancy. Any major changes in treatment should be made long before conception so that the effects of the new treatment can be assessed before pregnancy. The lowest effective dosage should be established for the appropriate drug as monotherapy. In some cases, seizure control can be maintained only with valproate. Low dose valproate (<800-1000 mg/day) may be no more harmful to the fetus than other drugs, and this dosage controls seizures in many patients. ${ }^{10} 1823$

\section{How do I monitor treatment and alter drug doses?}

Maternal plasma concentrations of antiepileptic drugs decline as pregnancy progresses. This may reduce seizure control and fetal drug exposure. ${ }^{9}$ Enhanced drug elimination due to induction of metabolising enzymes is the most common mechanism (box 2). This occurs with drugs metabolised through the cytochrome P450 system, such as phenytoin and phenobarbital, but it is even more

Malformation associated with monotherapy with different antiepileptic drugs

\begin{tabular}{|c|c|c|c|c|c|}
\hline \multirow[b]{2}{*}{ Register } & \multirow{2}{*}{$\begin{array}{l}\text { Total number of } \\
\text { pregnancies }\end{array}$} & \multicolumn{4}{|c|}{ Drug } \\
\hline & & Valproate & Carbamazepine & Lamotrigine & Phenobarbital \\
\hline Swedish register study ${ }^{25}$ & 1398 & $9.7 \%(268)$ & $4.0 \%(703)$ & - & - \\
\hline Finnish national drug prescription registry ${ }^{2}$ & 1231 & $10.6 \%(263)$ & $2.7 \%(805)$ & - & - \\
\hline UK epilepsy and pregnancy register ${ }^{18}$ & 3607 & $6.2 \%(715)$ & $2.2 \%(900)$ & $3.2 \%(647)$ & - \\
\hline Prospective international lamotrigine registry ${ }^{26}$ & 802 & - & - & $2.9 \%(802)$ & - \\
\hline $\begin{array}{l}\text { North American epilepsy and pregnancy } \\
\text { registry }{ }^{2427-29}\end{array}$ & & $10.7 \%(149)$ & $2.5 \%(873)$ & $2.7 \%(564)$ & $6.5 \%(77)$ \\
\hline
\end{tabular}

Values are reported percentage of malformations (number of exposed).

The registries summarised use different methodologies, have different criteria for malformations, and assess different populations. Malformation rates should therefore not be compared across studies. They are all non-randomised observational studies and do not provide class I evidence. 
pronounced for lamotrigine and possibly oxcarbazepine, drugs eliminated through glucuronidation. ${ }^{9}$ Lamotrigine plasma concentrations can decline during gestation to $30 \%$ or less of prepregnancy values, with subsequent breakthrough seizures. ${ }^{35-38}$ Similar but more limited data exist for oxcarbazepine. ${ }^{39}{ }^{40}$ However, women vary greatly in the way that pregnancy affects drug concentrations (box 2). Monitoring maternal drug concentrations is therefore recommended, particularly lamotrigine and oxcarbazepine, although evidence for the effectiveness of such monitoring in general is lacking. Ideally, one or two measurements should be obtained before pregnancy to record the patient's optimal serum concentration. Sampling once each trimester is often recommended, but more frequent sampling could be justified for lamotrigine. A pronounced decline during pregnancy might, as in our case, prompt a dose adjustment, especially if the patient has been sensitive to changes in drug concentrations before pregnancy. In settings where such monitoring is unavailable, dosage adjustments are based on clinical grounds alone, so closer clinical follow-up might be warranted. The importance of meticulous compliance should be stressed. An increase in dosage should be considered early if there is a trend for deterioration in seizure control, especially for drugs that are known to be affected by pregnancy.

\section{Preconception planning}

Young women with epilepsy need pregnancy related counselling. ${ }^{41}$ Box 3 lists the problems that should be discussed. This task may fall to the treating doctor or the patient may be referred to an appropriate centre. Because around half of pregnancies are unplanned, these problems should be brought up well before pregnancy is contemplated. Box 4 outlines strategies to optimise the treatment of epilepsy in patients seeking advice before pregnancy.

Unplanned pregnancies are often diagnosed later than five to 11 weeks. By then, the most sensitive period of fetal development has already passed, so it makes little sense to change the drug because of teratogenic risks (box 5). The woman should be told that inadvertent exposure to antiepileptic drugs is not an indication for therapeutic abortion. Counselling is as important here as it is before pregnancy, and it usually helps the woman to gain a realistic perspective of the risks. The problems listed in box 3 also apply in unplanned pregnancies.

\section{Box $2 \mid$ Mechanisms for altered drug concentrations in} pregnancy

- Enhanced metabolic elimination through enzyme induction (most important mechanism, relevant for drugs that are metabolised)

- Impaired drug absorption (for example, caused by hyperemesis gravidarum)

- Physiologically increased plasma volume

- Decreased drug binding to plasma proteins (relevant for highly bound drugs such as phenytoin and valproate; this does not alter drug effects, but total drug concentrations may be misleading-measure unbound concentrations if phenytoin orvalproate are monitored)

- Increased renal elimination
Box 3 | Problems to discuss in prepregnancy counselling of women with epilepsy

Genetic counselling including risk of inheriting epilepsy

- Risk is low in most types of epilepsy

Pharmacokinetic interactions between hormonal contraceptives and antiepileptic drugs

- Enzyme inducing antiepileptic drugs reduce the efficacy of steroidal contraceptives

- Contraceptives containing ethinylestradiol reduce concentrations of lamotrigine and possibly valproate

Expected course of epilepsy and risk of seizures

- The frequency of seizures is essentially the same as before pregnancy, but drug doses need to be increased in some patients to maintain adequate seizure control

The expected course of pregnancy and delivery

- Obstetric outcome is usually uneventful and caesarean section is needed only in the most difficult cases

Fetal and maternal risks associated with seizures

- Tonic-clonic seizures carry risks to the mother and fetus and should be avoided

Potential fetal risks associated with antiepileptic drugs

- Drugs can slightly increase the occurrence of

malformations

-Valproate should be avoided unless it is the only drug to give satisfactory seizure control

Possibilities and limitations of prenatal diagnostic test

- Expert ultrasonography can detect most neural tube defects and about two thirds of other major anomalies

- This facility may not be available everywhere

Principles of drug treatment of epilepsy during pregnancy

- Drugs should be given as monotherapy, at the lowest effective dosage

- Good compliance with treatment is essential

- Serum concentrations of drugs should be measured at least once each trimester

Folate prophylaxis

- Up to $5 \mathrm{mg} /$ day of folate-from before conception to the end of the first trimester-is usually recommended to all women taking antiepileptic drugs

Although evidence is lacking for the effectiveness of folic acid in preventing teratogenicity induced by antiepileptic drugs, and the appropriate dosage is still debated,,$^{42}$ many guidelines recommend that women taking such drugs should take up to $5 \mathrm{mg}$ of folate a day from before conception. ${ }^{43-46}$

\section{Obstetric management and breastfeeding}

Because of the definite, albeit small, risk of teratogenic effects of antiepileptic drugs, women taking these drugs should be offered prenatal diagnostics. Using modern targeted ultrasonography, almost all neural tube defects can be diagnosed at 12-22 weeks of pregnancy. ${ }^{46}$ Most of the other major structural abnormalities can also be detected, but the use of prenatal diagnostics depends on ethical issues and local legislation.

Extra obstetric follow-up is not needed in seizurefree patients, as their risk of common obstetric complications is not increased. ${ }^{313}$ Those with seizures in pregnancy have a risk of seizures in labour, ${ }^{8}$ so delivery should take place in an appropriately equipped unit.

Breast feeding is generally encouraged, ${ }^{43}$ although 
Box 4 | Optimising treatment of epilepsy in patients seeking advice before pregnancy

- Confirm diagnosis of epilepsy and reassess indication for treatment with antiepileptic drugs

- Consider gradually withdrawing drugs before conception if epilepsy is in remission and the risk of recurrence is low; the woman is aware of the risk and consequences of recurrence; and there is enough time before conception to ascertain whether epilepsy remains in remission after withdrawa

- Select the most appropriate antiepileptic drug for the patient's type of epilepsy; avoid valproate if equally effective alternatives are available

- Changing drugs in a woman who is seizure free is seldom justified except in the case of valproate

- Aim at monotherapy with the lowest effective dosage

- Document the patient's optimal drug concentration before pregnancy

relatively high drug concentrations have occasionally been reported in children of mothers treated with some drugs, such as phenobarbital, ethosuximide, and lamotrigine. ${ }^{47}$ Mothers taking these drugs should be told about the possibility of drug effects on the neonate but not generally advised against breast feeding.

\section{Conclusions}

Women with epilepsy need counselling to optimise treatment before conception. Antiepileptic drugs are usually needed to control seizures despite their indisputable, albeit low, risks to the fetus. Most patients will have uneventful pregnancies and deliveries, provided adequate care and facilities are available. Our scenario is an example of good and timely practice.

Contributors: TT and VH contributed equally with individual sections to the first draft, which was prepared by TT. TT and VH jointly revised and finalised the manuscript.

Funding: None.

Competing interests: TT has received speaker's honoraria or research grants (or both) from Eisai, GlaxoSmithKline, Janssen-Cilag, Novartis, Pfizer, Sanofi-Aventis, and UCB.

Provenance and peer review: Not commissioned; externally peer reviewed

1 Viinikainen K, Heinonen S, Eriksson K, Kalviainen R. Communitybased, prospective, controlled study of obstetric and neonatal outcome of 179 pregnancies in women with epilepsy. Epilepsia 2006;47:186-92.

2 Artama M, Auvinen A, Raudaskoski T, Isojärvi J. Antiepileptic drug use of women with epilepsy and congenital malformations in offspring. Neurology 2005;64:1874-8.

Box 5 | Optimising treatment of epilepsy in patients who are already pregnant

- Alleviate fears of a patient with unplanned pregnancy (box 3 )

- Withdrawing or changing antiepileptic drugs is rarely justified if seizures are well controlled. Risks probably outweigh potential gains

- Monitor treatment more closely than normal; the frequency of clinical visits depends on seizure control and other factors, but once a trimester is standard; monitor drug concentrations of lamotrigine in particular and possibly oxcarbazepine

- Adjust drug doses to optimise treatment in patients who have tonic-clonic seizures during pregnancy

- Dosage adjustments may also be justified in patients with an increase in other seizure types and in patients who have a pronounced decline in drug concentrations

- Reassure the patient that with adequate treatment the neurological and obstetric risks are low
3 Katz O, Levy A, Wiznitzer A, Sheiner E. Pregnancy and perinatal outcome in epileptic women: a population-based study. J Matern Fetal Neonatal Med 2006;19:21-5

4 Bardy AH. Incidence of seizures during pregnancy, labor and puerperium in epileptic women: a prospective study. Acta Neurol Scand 1987;75:356-60.

5 Gjerde IO, Strandjord RE, Ulstein M. The course of epilepsy during pregnancy: a study of 78 cases. Acta Neurol Scand 1988;78:198-205.

6 Tomson T, Lindbom U, Ekqvist B, Sundqvist A. Epilepsy and pregnancy: a prospective study of seizure control in relation to free and total plasma concentrations of carbamazepine and phenytoin. Epilepsia 1994:35:122-30.

7 Sabers A, Rogvi-Hansen B, Dam M, Fischer-Rasmussen W, Gram L, Hansen M, et al. Pregnancy and epilepsy: a retrospective study of 151 pregnancies. Acta Neurol Scand 1998;97:164-70.

8 The EURAP Study Group. Seizure control and treatment in pregnancy. Observations from the EURAP epilepsy pregnancy registry. Neurology 2006;66:354-60.

9 Tomson T, Battino D. Pharmacokinetics and therapeutic drug monitoring of newer antiepileptic drugs during pregnancy and the puerperium. Clin Pharmacokinet 2007;46:209-19.

10 Adab N, Kini U, Vinten J, Ayres J, Baker G, Clayton-Smith J, Coyle H, et al. The longer term outcome of children born to mothers with epilepsy. J Neurol Neurosurg Psychiatry 2004;75:1575-83.

11 Lipka K, Bulow HH. Lactic acidosis following convulsions. Acta Anaesthesiol Scand 2003:47:616-8.

12 Hiilesmaa VK, Bardy A, Teramo K. Obstetric outcome in women with epilepsy. Am J Obstet Gynecol 1985;152:499-504.

13 Richmond JR, Krishnamoorthy P, Andermann E, Benjamin A. Epilepsy and pregnancy: an obstetric perspective. Am J Obstet Gynecol 2004;190:371-9.

14 Hiilesmaa VK. Pregnancy and birth in women with epilepsy. Neurology 1992;42(suppl 5):8-11.

15 Fried S, Kozer E, Nulman I, Einarson TR, Koren G. Malformation rates in children of women with untreated epilepsy: a meta-analysis. Drug Saf 2004;27:197-202.

16 Tomson T, Perucca E, Battino D. Navigating toward foetal and maternal health: the challenge of treating epilepsy in pregnancy. Epilepsia 2004;45:1171-5.

17 Perucca E. Birth defects after prenatal exposure to antiepileptic drugs. Lancet Neurol 2005;4:781-6.

18 Morrow I, Russell A, Guthrie E, Parsons L, Robertson I, Waddell R, et al. Malformation risks of antiepileptic drugs in pregnancy: a prospective study from the UK epilepsy and pregnancy register. J Neurol Neurosurg Psychiatry 2005;77:193-8.

19 Kaneko S, Battino D, Andermann E, Wada K, Kan R, Takeda A, et al. Congenital malformations due to antiepileptic drugs. Epilepsy Res 1999;33:145-58.

20 Samren EB, van Duijn CM, Christiaens GC, Hofman A, Lindhout D. Antiepileptic drug regimens and major congenital abnormalities in the offspring. Ann Neurol 1999;46:739-46.

21 Holmes LB, Harvey EA, Coull BA, Huntington KB, Khoshbin S, Hayes AM, et al. The teratogenicity of anticonvulsant drugs. N Engl/ Med 2001;344:1132-8

22 Kaaja E, Kaaja R, Hiilesmaa V. Major malformations in offspring of women with epilepsy. Neurology 2003;60:575-9.

23 Vajda FJ, O’Brien TJ, Hitchcock A, Graham J, Cook M, Lander C, et al. Critical relationship between sodium valproate dose and human teratogenicity: results of the Australian register of anti-epileptic drugs in pregnancy. I Clin Neurosci 2004:11:854-8.

24 Holmes LB, Wyszynski DF, Baldwin EJ, Habecker E, Glassman LH, Smith CR. Increased risk for non-syndromic cleft palate among infants exposed to lamotrigine during pregnancy. Birth DefRes Clin Mol Teratol 2006;76:318

25 Wide K, Winbladh B, Kallen B. Major malformations in infants exposed to antiepileptic drugs in utero, with emphasis on carbamazepine and valproic acid: a nation-wide, population-based register study. Acta Paediatr 2004;93:174-6.

26 Cunnington M, Ferber S, Quartney G; International Lamotrigine Pregnancy Registry Scientific Advisory Committee. Effect of dose on the frequency of major birth defects following fetal exposure to lamotrigine monotherapy in an international observational study. Epilepsia 2007;48:1207-10

27 Wyszynski DF, Nambisan M, Surve T, Alsdorf RM, Smith CR, Holmes LB. Increased rate of major malformations in offspring exposed to valproate during pregnancy. Neurology 2005;64:961-5.

28 Hernandez-Diaz S, Smith CR, Wyszynski DF, Holmes LB. Risk of major malformations among infants exposed to carbamazepine during pregnancy. Birth Defects Res Clin Mol Teratol 2007;79:357.

29 Holmes LB, Wyszynski DF, Lieberman E. The antiepileptic drug pregnancy registry: a 6-year experience. Arch Neurol 2004;61:673-8.

30 Tomson T, Battino D. Teratogenicity of antiepileptic drugs: state of the art. Curr Opin Neurol 2005;18:135-40.

31 Adab N, Tudur SC, Vinten J, Williamson PR, Winterbottom JB. Common antiepileptic drugs in pregnancy in women with epilepsy. Cochrane Database Syst Rev 2004; (3):CD004848. 
32 Vinten J, Adab N, Kini U, Gorry J, Gregg J, Baker G. Neuropsychological effects of exposure to anticonvulsant medication in utero. Neurology 2005;64:949-54

33 Gaily E, Kantola-Sorsa E, Hiilesmaa V, Isoaho M, Matila R, Kotila $\mathrm{M}$, et al. Normal intelligence in children with prenatal exposure to carbamazepine. Neurology 2004;62:28-32.

34 Eriksson K, Viinikainen K, Monkkonen A, Aikia M, Nieminen, $P$, Heinonen S, et al. Children exposed to valproate in utero: population-based evaluation of risks and confounding factors for long-term neurocognitive development. Epilepsy Res 2005;65:189-200.

35 Tran TA, Leppik IE, Blesi K, Sathanandan ST, Remmel R. Lamotrigine clearance during pregnancy. Neurology 2002;59:251-5.

36 Pennell PB, Newport DJ, Stowe ZN, Helmers SL, Montgomery JQ, Henry TR. The impact of pregnancy and childbirth on the metabolism of lamotrigine. Neurology 2004;62:292-5.

37 De Haan GJ, Edelbroek P, Segers J, Engelsman M, Lindhout D, DevileNotschaele M, et al. Gestation-induced changes in lamotrigine pharmacokinetics: a monotherapy study. Neurology 2004;63:571-3.

38 Petrenaite V, Sabers A, Hansen-Schwartz I. Individual changes in lamotrigine plasma concentrations during pregnancy. Epilepsy Res 2005;65:185-8.

39 Mazzucchelli I, Onat FY, Özkara C, Atakli D, Specchio LM, Neve AL, et al. Changes in the disposition of oxcarbazepine and its metabolites during pregnancy and the puerperium. Epilepsia 2006;47:504-9.

40 Christensen J, Sabers A, Sidenius P. Oxcarbazepine concentrations during pregnancy: a retrospective study in patients with epilepsy. Neurology 2006;67:1497-9.

41 Kälviäinen R, Tomson T. Optimizing treatment of epilepsy during pregnancy. Neurology 2006;67(suppl 4):S59-63.

42 Moore JL. The significance of folic acid for epilepsy patients. Epilepsy Behav 2005;7:172-81.

43 Stokes T, Juarez-Garcia A, Camosso-Stefinovic J, Baker R. Clinical guidelines and evidence review for the epilepsies: diagnosis and management in adults and children in primary and secondary care. London: Royal College of General Practitioners, 2004.

44 Crawford P. Best practice guidelines for the management of women with epilepsy. Epilepsia 2005;46(suppl 9):117-24.

45 Breen DP, Davenport RJ. Teratogenicity of antiepileptic drugs. BMJ 2006;333:615-6.

46 Kooper AJ, de Bruijn D, van Ravenwaaij-Arts CM, Faas BH, Creemers IW, Thomas CM, et al. Fetal anomaly scan potentially will replace routine AFAFP assays for the detection of neural tube defects. Prenat Diagn 2007;27:29-33.

47 Tomson T. Gender aspects of pharmacokinetics of new and old AEDs: pregnancy and breast-feeding. Ther Drug Monit 2005;27:718-21.

\section{LESSON OF THE WEEK}

\section{An unusual cause of jaundice}

\author{
PJ D Owen, ${ }^{1}$ A Baghomian, ${ }^{2}$ J H Lazarus', A J Godkin³
}

Centre for Endocrine and Diabetes Sciences, University Hospital of Wales, Cardiff

${ }^{2}$ Department of Gastroenterology, Glan Clwyd Hospital, Rhyl

${ }^{3}$ Department of Integrated Medicine, University Hospital of

Wales, Cardiff

pennyowen74@yahoo.co.uk

BMJ 2007;335:773-4

doi: 10.1136/bmj.39247.452141.BE

\section{Patients with jaundice of unknown cause need their thyroid function tested to exclude an underlying thyroid problem}

We report on a patient with Graves' thyrotoxicosis, whose presentation with jaundice and hepatic dysfunction led to unnecessary investigations and a delay in management. We suggest patients with jaundice of unknown cause should have thyroid function tests performed as a part of their routine investigation.

\section{Case report}

A 36 year old labourer was referred to the gastroenterology department at his local hospital with a three month history of general malaise, myalgia, and painless jaundice. He reported a $25 \mathrm{~kg}$ weight loss and a change of bowel habit with pale diarrhoea and steatorrhoea, passing stools up to 20 times daily, with some darkening of his urine.

He had no medical history of note, was a nonsmoker, consumed around 5 units of alcohol a week, and lived with his wife and teenage daughter. He had not travelled abroad recently and had had no occupational exposure to hepatitis or hepatotoxic chemicals or drugs. In addition, he had not received any blood products, undergone body piercings, or experienced any previous episodes of jaundice.

On clinical examination he was markedly cachetic and icteric (fig 1), with no signs of chronic liver disease or tattoos evident. He had no fever and a pulse rate of 78 beats/min, blood pressure of $134 / 78 \mathrm{~mm} \mathrm{Hg}$, respiratory rate of 14 breaths/min, and BMI of 23.9. We detected no lymphadenopathy or testicular atrophy or organomegaly on abdominal examination.

Results of full blood count and tests for urea, electrolytes, and random glucose were all within normal limits. Inflammatory markers were raised with the erythrocyte sedimentation rate of $47 \mathrm{~mm}$ in the first hour and C reactive protein of $52 \mathrm{mg} / \mathrm{l}$. Results of routine hepatic function tests were 581 (normal range 1-22) $\mu \mathrm{mol} / \mathrm{l}$ for bilirubin, 184 (30-115) U/l for alkaline phosphatase, 26 (5-48) U/1 for $\gamma$-glutamyltransferase, 146 (5-40) U/1 for alanine aminotransferase, 69 (5-45) U/l for aspartate aminotransferase, and $33 \mathrm{~g} / \mathrm{l}$ (35-50) for albumin. Results of clotting and coagulation studies, and hepatitis investigations were normal, and autoantibody testing showed positive smooth muscle antibody of low titre (1:40), with negative results for other autoantibodies (IgA antitransglutaminase, antinuclear, antidouble stranded DNA, antineutrophil cytoplasmic, and antimitochondrial antibodies).

Abdominal ultrasonography showed normal liver, biliary tree, and gall bladder with no evidence of biliary obstruction; computerised tomography of the abdomen also showed normal liver size and texture. Percutaneous liver biopsy showed evidence of cholestasis, and subsequent magnetic resonance cholangiopancreatography found no abnormality in the bile and pancreatic ducts.

Because of his unresolving jaundice and ongoing hepatic dysfunction with an undefined cause the initial medical team investigating his condition considered liver transplantation. He was subsequently referred to the gastroenterology department at our tertiary centre for a further opinion.

On review at our hospital by the gastroenterologists 